In most cases admitted to the programme the psychological debriefing represents an immediate component which was neglected after traumatic exposure which permits secure retrieval of memory information and which facilitates the processing of the same.

Busltril. W.. Turnbuli., G. J.. Neal. L. A., et al (1995) Incorporating psychological debriefing techniques within a brief group psychotherapy programme for the treatment of posttraumatic stress disorder. British Journal of Psychiatry, 167, $495 \cdot 502$.

Ticehurst House Hospital

G. J. Turnbull

East Sussex TN5 $7 \mathrm{HU}$

SIR: Comparisons between the group treatment approach described by Busuttil et al (1995) for war veterans and the well documented Koach project (Solomon et al, 1992) highlight important treatment and research issues. The Koach project was a residential group treatment approach for PTSD developed to treat Israeli war veterans. Based on a behavioural model of PTSD, a central treatment component involved confronting avoidance using a number of behavioural techniques but there was "a deliberate refusal ... to allow working through of past war experiences" (Shalev et al, 1992; p. 214). While subjective appraisal by therapists and the 41 participants was highly positive and therapeutic gains were shown in reducing phobias and improving social functioning, no improvement was noted in the symptoms of PTSD and at two year follow up the treatment group had a worse outcome than a no-treatment control group.

The Busuttil study in comparison incorporated no behavioural procedures directly aimed at anxiety habituation and extinction and the focus of therapy was the "psychological debriefing" or information processing component. It is necessary to acknowledge possible group differences, the methodological flaws of the studies, the possibility that the Israeli study may not have ensured that habituation of anxiety accompanied exposure and that debriefing did allow for some habituation of anxiety. Nonetheless, the high success rate in reducing PTSD symptoms such that $85.3 \%$ no longer satisfied the criteria for diagnosis of PTSD is in marked contrast to the Israeli findings.

It has been questioned whether informationprocessing models of PTSD add anything to Pavlovian models of extinction (Hacker-Hughes \& Thompson, 1994). Richards \& Rose (1991) found in a study which looked at imaginal exposure and in vivo exposure, that "where in vivo exposure was tried first, it was either ineffective or only partially effective" (p. 839). In a study of an implosion treatment with rape survivors, Foa et al (1995) also present findings showing that successful outcome was associated with changes in subjects' rape narratives suggestive of information processing of the event. In the same article Foa quotes other authors who conclude that for trauma survivors, the need to make sense of their traumatic experience is of vital importance.

Much of the treatment literature in PTSD has a behavioural or cognitive-behavioural bias. Such approaches have proved valuable but other theoretical models may have the potential to enhance both the understanding of PTSD and therapeutic effectiveness.

Busuttil, W., Turnbull. G. J., Neal., L. A., et al (1995) Incorporating psychological debriefing techniques within a brief group psychotherapy programme for the treatment of posttraumatic stress disorder. British Journal of Psychiatry. 167, 495-502.

Foa, E. B., Molnar, C. \& Cashman, L. (1995) Change in rape narratives during exposure therapy for post-traumatic stress disorder. Journal of Traumatic Stress, 8, 675-691.

Hacker-Hughes, J. G. H. \& Thompson, J. (1994) Post-traumatic stress disorder: An evaluation of behavioural and cognitive behavioural interventions and treatment. Clinical Psychology and Psychotherapy, 1, 125-142.

RichardS, D. A. \& RoSe, J. S. (1991) Exposure therapy for post-traumatic stress disorder. British Journal of Psychiatry. $158,836-840$.

Shalev, A., Spiro, S. E., Solomon, Z., et al (1992) The effectiveness of the Koach project: positive clinical impressions and negative psychometric outcomes; positive clinical impressions: 1. Therapists evaluations. Journal of Traumatic Stress, 5, 205 217.

Solomon, Z., Bleich, A., Shoham, S., et al (1992) The Koach project for treatment of combat related PTSD: rationale, aims and methodology. Journal of Traumatic Stress, 5, 175-195.

Kings Lynn, Norfolk PE33 9PA

A. BusutTIL

\section{Psychiatric morbidity in rural $v$. urban regions}

SIR: Mumford et al (1996) showed that women in Chitral, Pakistan, suffered surprisingly more anxiety and depressive disorders than their counterparts in Western countries. This finding contradicts the common belief that people who live in remote rural areas of the globe, being exempt from the pressure of civilization, lead relatively stress-free lives. However, it is not so surprising when one considers that socioeconomic and political changes have negatively impinged on the livelihood of rural people and aggravated their powerlessness in many developing countries nowadays (Desjarlais et al, 1995). 